\title{
Lateral Asymmetry in the Hippocampal Response to the Remoteness of Autobiographical Memories
}

\author{
Eleanor A. Maguire and Christopher D. Frith \\ Wellcome Department of Imaging Neuroscience, Institute of Neurology, University College London, London WC1N 3BG, United Kingdom
}

\begin{abstract}
The time scale of hippocampal involvement in retrieving memories, particularly those more remote, is still a matter of debate. Some propose that the hippocampus is not involved in the retrieval of remote memories, whereas others assert that it is necessary for memory retrieval in perpetuity. Functional magnetic resonance imaging was used to examine the effect of remoteness on the neural basis of memory. We used a parametric event-related random-effects design in a large group of subjects to overcome some of the limitations of previous neuroimaging studies. We found that the hippocampi were significantly active during the retrieval of autobiographical memories. Notably, the two hippocampi diverged in their responses to remoteness. The right hippocampus showed a temporal gradient, decreasing in activity the more remote the autobiographical memories. No such effect was apparent in the left hippocampus, suggesting its invariant involvement in remembering autobiographical events throughout the lifespan. The dorsal amygdalas showed a temporal gradient similar to the right hippocampus, but emotional valence and intensity were not directly associated with changes in activity. The current results indicate that consideration of lateral asymmetry may help to broaden the scope of theoretical interpretations concerning hippocampal involvement in remote memory.
\end{abstract}

Key words: fMRI; hippocampus; temporal gradient; consolidation; episodic memory; amygdala

\section{Introduction}

It is widely accepted that the hippocampus plays a pivotal role in the formation and consolidation of memories for the spatially and temporally specific (autobiographical) events that comprise our everyday lives (Scoville and Milner, 1957). The time scale of hippocampal involvement in these processes, and whether it is necessary for the retrieval of memories once they become more remote, remains an open question. Two prominent theories, posited primarily on the basis of patient data, present differing views on the role of the hippocampus in remote memory. The consolidation theory asserts that the hippocampus is required for memory formation and initial consolidation before neocortical areas assume the responsibility for memory storage and retrieval independent of the hippocampus (Squire, 1992). In contrast, the multiple-trace theory (MTT) contends that each autobiographical memory has a permanent trace in the hippocampus (Nadel and Moscovitch, 1997; see also, Sanders and Warrington, 1971; Murray and Bussey, 2001). Reconciling these theoretical positions from patient data alone is problematic. The length of retrograde amnesia (RA), or loss of memories that were formed before lesion occurrence, has been taken as key evidence in deducing the hippocampal role. However, even with apparently similar selective bilateral hippocampal lesions, patients have been reported with a relatively short RA (Zola-Morgan et al., 1986), but others

Received Nov. 4, 2002; revised March 31, 2003; accepted March 31, 2003.

This work was supported by the Wellcome Trust. We thank Catriona Good for examination of structural scans, Alexa Morcom for assistance with subject recruitment, and Rik Henson, Uta Noppeney, and Will Penny for useful discussions.

Correspondence should be addressed to Dr. Eleanor Maguire, Wellcome Department of Imaging Neuroscience, Institute of Neurology, University College London, 12 Queen Square, London WC1N 3BG, UK. E-mail: e.maguire@fil.ion.ucl.ac.uk.

Copyright $\odot 2003$ Society for Neuroscience $\quad$ 0270-6474/03/235302-06\$15.00/0 had RA stretching back over decades (Cipolotti et al., 2001; for a review of hippocampal amnesia, see Spiers et al., 2001).

Functional neuroimaging in healthy subjects offers a complementary means to examine the effect of remoteness on the neural basis of memory. However, there have been a limited number of studies examining remote memory (Conway et al., 1999; Stark and Squire, 2000; Maguire et al., 2001a; Ryan et al., 2001; Haist et al., 2001; Niki and Luo, 2002). The findings from these studies are consistent, with no reliable evidence of modulation in the activity of the hippocampus in response to the remoteness of memories ${ }^{a}$ [The hippocampus is defined here as CA1-CA4 and dentate gyrus, in line with Amaral (1999)]. These studies appear to be problematic for the consolidation theory. However, there are a number of problems with the studies that cast doubt on this conclusion. All but one study used a block rather than an eventrelated design. In a block design, the activity associated with a specific memory event is not examined, instead activity is averaged over many events. This makes it impossible to distinguish specific stimulus-related effects (e.g., the remoteness of each memory) from state-related changes in activity unrelated to the processing of specific stimuli but which are tonically maintained across a block of trials (Rugg, 1998; Otten et al., 2002). Thus, transient event-related effects (such as remoteness) may be obscured. Furthermore, the studies have been limited in the numbers of subjects tested and memories sampled.

The aim of this study was to examine the hippocampal response to both autobiographical events and public events dating from decades ago through the present day, overcoming some of

\footnotetext{
${ }^{a}$ In two studies, the hippocampus was not active during the retrieval of either recent or remote memories, making specific inferences about the hippocampus difficult (Conway et al., 1999; Niki and Lu0, 2002). In another study, the hippocampus was active for recent memories, and it was suggested that there was evidence of a temporal gradient in its activity. However, on direct examination, there were no statistically reliable differences between recent and remote memories in terms of hippocampal activity (Haist et al., 2001).
} 
the limitations of previous studies. We included the two memory types because there continues to be debate about whether the hippocampus is concerned with all forms of explicit memory (Squire and Zola-Morgan, 1997) or most particularly with autobiographical event memory (Nadel and Moscovitch, 1997; Maguire, 2001). Each recollection was modeled as a single event, permitting the use of a parametric design and eliminating the need for their arbitrary categorization as recent or remote, or the need to average over many events. We included a large number of subjects and a large number of events of each memory type. In addition, ratings of emotional valence and intensity were included for each memory event and explicitly modeled.

\section{Materials and Methods}

Subjects. All values reported are means \pm SD. The sample comprised 24 participants with a mean age of $53.58 \pm 22.08$ years (range, 23-80). Twelve subjects were young (mean age, $32.42 \pm 4.21$; range, 23-39; 6 males, 6 females). Twelve subjects were older (mean age $74.75 \pm 4.92$; range, 67-80; 6 males, 6 females). There were two left-handed males, one young and one older. All subjects were healthy and had been screened to exclude neurological, psychiatric, or systemic conditions or medication that might affect brain function. In addition, the structural MRI scan of each subject was examined by a neuroradiologist to rule out pathology. The mean number of years of education beyond the age of 16 years was $5.5 \pm 3.43$ for the young subjects and $4.58 \pm 2.61$ for the older subjects (the difference was not significant; $p=0.47$ ). All subjects were high functioning (mostly university-educated), autonomous communitydwellers. The older subjects, although retired, were all active in cultural pursuits, in continuing education, or with responsibilities in various associations. Given the auditory nature of the experiment, the hearing of each subject was checked before scanning to ensure that the tasks could be performed in the scanning context. All subjects gave written informed consent in accordance with the local medical ethics committee.

Image acquisition and data analysis. Data were acquired using a 2 tesla Magnetom VISION (Siemens GmbH, Erlangen, Germany) MRI system. Contiguous multislice $\mathrm{T}_{2}{ }^{*}$-weighted functional MRI (fMRI) images were obtained (echo time $=40 \mathrm{msec}$ ) with echo-planar imaging (EPI), whole head: 32 slices, each $3 \mathrm{~mm}$ thick, $3.1 \mathrm{sec}$ per volume. A structural MRI scan using a standard three-dimensional $\mathrm{T}_{1}$-weighted sequence was acquired from each subject. Functional images were processed and analyzed using Statistical Parametric Mapping (SPM99; Wellcome Department of Imaging Neuroscience, London, UK) (Friston et al., 1995). Briefly, the first five volumes were discarded to allow for $\mathrm{T}_{1}$ equilibration effects. Images were realigned to correct for interscan movement, normalized to a standard EPI template based on the Montreal Neurological Institute reference brain and resampled to $3 \times 3 \times 3 \mathrm{~mm}^{3}$. The $\mathrm{T}_{1}$ structural volume was coregistered with the mean realigned EPI volume and normalized with the same deformation parameters. The normalized images were smoothed with an isotropic $8 \mathrm{~mm}$ full-width half-maximum Gaussian kernel to accommodate intersubject anatomical variability. The hemodynamic response to each stimulus event (statement plus response) was modeled by a canonical hemodynamic response function and its first-order temporal derivative. The age (in years) of each autobiographical event memory and each public event memory and the ratings of both valence and intensity (see below) for each of these memories were included as covariates of interest (modeled linearly). The six headmovement parameters were included as confounding factors. First-level linear contrasts of parameter estimates for each subject were taken to the second level, and a random-effects analysis was performed. A threshold of $p<0.001$ (uncorrected for multiple comparisons) was used throughout. Activations involving clusters of more than three contiguous voxels were interpreted.

Study materials and procedure. There is an ongoing debate about how to assess remote memory (Kapur, 1999). Here we used a paradigm similar to that reported previously (Maguire et al., 2000, 2001a,b). This approach has resulted in robust and consistent hippocampal activations, an essential platform from which to examine the effects of remoteness on hippocampal activity. Several weeks before scanning, subjects were inter- viewed at length to check general and personal factual knowledge and to ascertain details of their personal event memories and knowledge of public events from the time they were $4-5$ years old to the present day. Only events that involved the precise recall of time and place of occurrence, were definitely recollected by the subject and not told to them by a third party, and were recalled with the most detail were considered for inclusion in the scanning experiment. The richness of details was scored in a manner similar to that described by Nadel et al. (2000), that is, by counting the number of details provided for each event. The memories selected for inclusion were those that were recalled with the most details (but excluding those events for which the details were of one particular type, e.g., for a memory involving a car, in which the details pertained only to the visual/perceptual aspects of the car, but with few details about the overall event involving the car). This approach also served to exclude event memories that had become semanticized (Moscovitch et al., 1999; Rosenbaum et al., 2001). There was no difference in the amount or richness of detail of recent and remote memories that were included in the scanning experiment. We were also careful to exclude public events that had clear autobiographical associations. The interview information was used to construct stimuli specific to each individual subject. Four memory types were included: autobiographical events, public events, autobiographical facts, and general knowledge. Because the issue of interest here concerns possible neural correlates of the remoteness of memories, only data pertaining to the first two memory types are reported, because they had specific spatiotemporal contexts (see Maguire and Frith, 2003, for data relating to the other memory types).

The stimuli comprised sentences presented auditorily during fMRI scanning; the task was to listen to a statement and verify if it was true or false by a key-press response. The true:false ratio was $3: 1$, with the false statements comprising obvious adjustments to genuine memories. Previous studies using a similar paradigm found that for event memories, this task caused subjects to recall the original events even during the false statements (Maguire et al., 2001a). There were 24 statements (scanning events) for each memory type. Each statement was between 3 and 4 sec long, and subjects responded up to a maximum of $8 \mathrm{sec}$ from statement onset. Across all memory conditions, statements were controlled for length of presentation, number of syllables, and frequency of proper nouns. Autobiographical and public events stimuli were matched for the age of the memories, with an even spread throughout the lifetime of a subject. Subjects rated each autobiographical event and each public event memory for emotional valence. The question asked was "How does thinking about this make you feel?"; subjects responded using a 7 point scale ranging from -3 (very unhappy) to +3 (very happy). They also rated the intensity of their feelings in relation to each memory event on a 7 point scale raging from 1 (low intensity) to 7 (high intensity). Examples of stimuli include an autobiographical event (You sang in the Requiem at St. Albans Abbey.) and a public event (The Stone of Scone was stolen from Westminster Abbey.).

\section{Results}

Aspects of the data from these subjects have been reported previously in relation to a different research question about memory and aging (Maguire and Frith, 2003). We now report novel analyses that speak directly to the current questions, namely the effects of remoteness and emotion on the neural basis of autobiographical and public event memories.

Subjects were debriefed after scanning using a simple procedure. They were asked to state what they had been thinking about when they heard the stimuli. They were also asked to say whether they thought about the previous interview during scanning or when hearing the stimuli. Subjects confirmed that for event memories, the task had caused them to recall the original event, typically with a subjective sense of re-experiencing it. None of the subjects reported thinking about the previous interview during scanning, and none reported any of the tasks to be more difficult than another. The mean reaction times for autobiographical events was $4053.08 \pm 356.7 \mathrm{msec}$ (mean $\pm \mathrm{SD}$ ) and for public 
events $4118.83 \pm 193.9 \mathrm{msec}$; the difference was not significant. For both young and older subjects, the most recent memories were within the 6 months before scanning. For young subjects, remote memories ranged from 17 to 35 years old, and in older subjects from 61 to 77 years old.

\section{Behavioral data}

Performance on the true/false task during scanning was very accurate and similar for both autobiographical events (23.17 \pm $0.68 / 24)$ and public events $(22.88 \pm 0.85 /$ 24). The mean emotional valence ratings for the two memory types were close to neutral, with the autobiographical events being slightly positive $(0.39 \pm 1.69)$ and the public events slightly negative $(-0.75 \pm 1.22)$; the range for both was -3 to +3 . The mean intensity ratings for the two memory types were also similar (autobiographical events, $3.89 \pm 1.87$; public events, $3.16 \pm 1.84)$, with a range from 1 to 7 for both. Across all memories, valence and intensity ratings were correlated with each other for both autobiographical ( $r=$ $0.2 ; p<0.0001)$ and public events $(r=$ $-0.45 ; p<0.0001)$. For autobiographical events, valence was correlated with the remoteness of memories $(r=-0.13$; $p<$

0.003 ) when between subjects and intensity effects were partialled out. This means more recent memories were regarded as more positive. Valence was also correlated with remoteness in the case of public events $(r=0.1 ; p<0.003)$, but in this instance recent memories were regarded as more negative. Intensity ratings did not correlate with remoteness for either memory type.

\section{fMRI data}

Direct comparisons between the two memory types showed that the left $(-21,-9,-18 ; z=5.33)$ and right $(21,-15,-15 ; z=$ 5.00) hippocampi (Fig. 1), medial frontal cortex (0,54, $0 ; z=$ $5.52)$, and retrosplenial cortex $(-3,-55,18 ; z=4.87)$ were more active during the retrieval of autobiographical compared with public events. The reverse contrast showed that the left superior temporal gyrus $(-57,-12,6 ; z=4.06)$ was more active for public than for autobiographical events.

A parametric effect of autobiographical memory remoteness was revealed in only two regions, the right hippocampus (18, $-21,-15 ; z=3.7)$ (Fig. $2 A)$ and the left dorsal amygdala $(-24$, $0,-18 ; z=3.47$ ) (Fig. $2 B$ ), with activity decreasing the more remote the autobiographical memories. The right dorsal amygdala showed a similar response just below threshold, but the left hippocampus did not show this effect even at a very liberal statistical threshold. We also defined two regions of interest $(10 \mathrm{~mm}$ spherical) in the left and the right hippocampi centered on the voxel of peak activation and statistically compared the mean time course of the voxels within the two regions directly (using MarsBar, Brett et al., 2002). This confirmed the significant laterality difference in response (two-tailed $p<0.05$ ). The parameter estimate (arbitrary units) at the peak voxel in the right hippocampus was 5.7 (between subjects SE, 1.0) and at the same voxel in the left hippocampus (only detectable at a very liberal threshold of $p<0.05$ uncorrected) was 2.6 (between subjects SE, 0.9). This clearly shows the much greater effect size for the parametric effect of memory remoteness in the right hippocampus.

Figure 3, $A$ and $B$, shows three-dimensional plots of the response to remoteness in one example young and one example old subject, respectively, at the peak right hippocampal voxel reported in the group analysis. The plots show that although clearly most active for recent memories with its activity decreasing with memory age, the right hippocampus remains active even for 30year-old memories (both plots). It is only when memories are older than $\sim 30$ years that the right hippocampus is deactivated (Fig. 3B).

No brain regions showed increasing activity the more remote the autobiographical memories. No decreases or increases in activity were associated with the remoteness of public event memories. In a previous study we found evidence of a temporal gradient in the right ventrolateral prefrontal cortex for autobiographical events (Maguire et al., 2001a). In the current study, this area showed the same temporal gradient effect but was subthreshold.

Ratings of emotional valence and intensity were not associated with increases or decreases in activity for either autobiographical or public event memories

\section{Discussion}

There are three key findings from our study. First, activity in the hippocampi was significantly increased while remembering autobiographical events compared with remembering public events. Second, the right and left hippocampi diverged with respect to their response to memory remoteness. The right hippocampus showed a temporal gradient, with its activity decreasing the more remote the autobiographical memories. In contrast, 

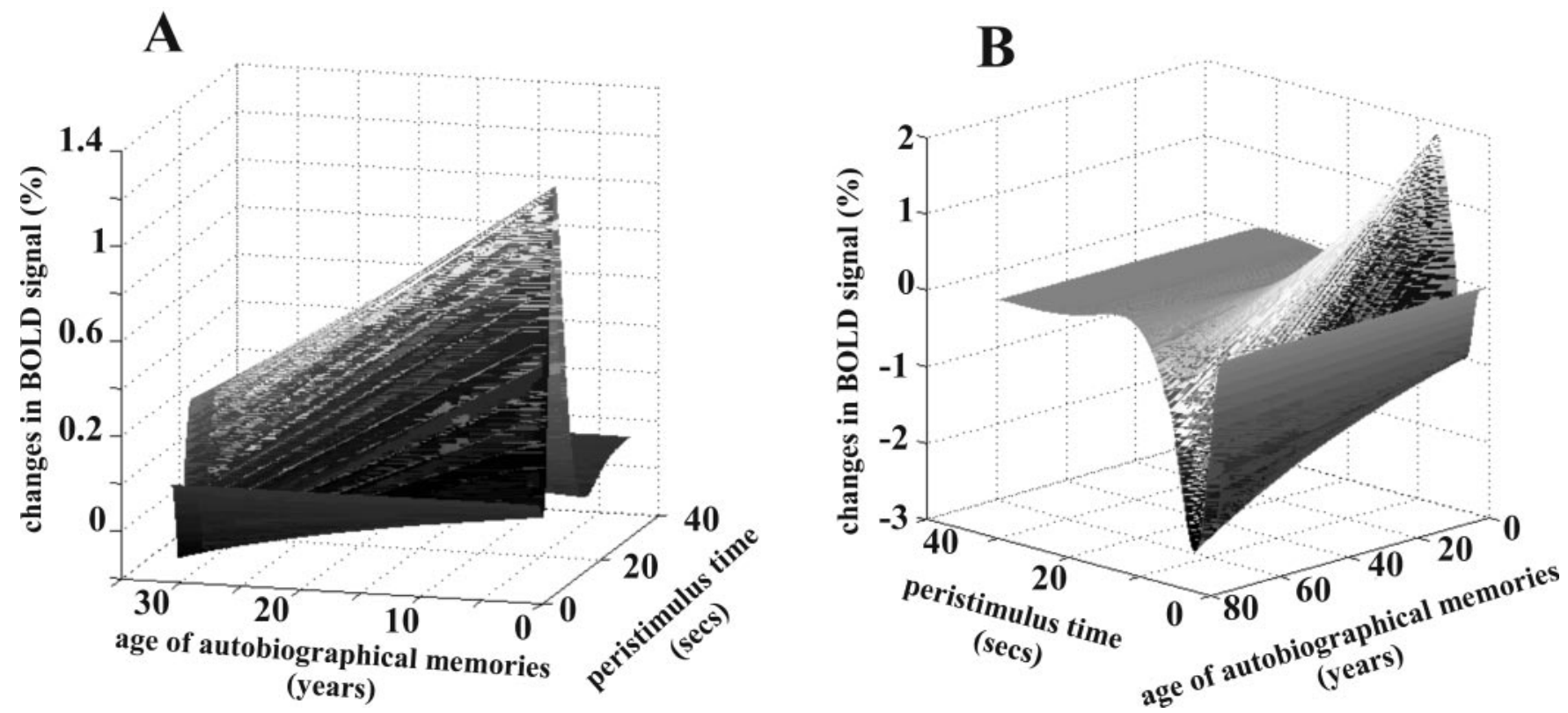

Figure 3. The hippocampal remoteness effect graphically represented. A three-dimensional plot of the response to remoteness at the peak voxel reported in the group analysis in an example young $(A)$, and an example older $(B)$ subject. The plots are shown in slightly different orientations to afford the optimal view of the temporal gradient in each case. BOLD, Blood-oxygen level dependent.

activity in the left hippocampus was not modulated by the remoteness of memories, suggesting invariant involvement in remembering autobiographical events throughout the lifespan. Third, emotional valence and intensity were not directly associated with changes in activity, although the dorsal amygdalas were observed to show a temporal gradient, decreasing in activity the more remote the autobiographical memories. Notably, the findings pertained to memory for autobiographical but not public events, although the two event types were recalled with similar detail, were matched for memory age, and required orientation to the past.

\section{Memory}

A preferential role for the hippocampus in the retrieval of autobiographical events compared with other memory types such as public events has been documented previously, and often with a left hippocampal preponderance (Maguire and Mummery, 1999; Maguire et al., 2000, 2001a,b; Burgess et al., 2001; Ryan et al., 2001; but see Fink et al., 1996). It may be that our larger number of subjects and memory events revealed previously undetected right hippocampal activity. However, half of the current subjects were in their 20s and 30s, and half were over the age of 65. Previously, left hippocampal activation was reported in young subjects during autobiographical memory retrieval, with bilateral hippocampal activation evident in older adults. Direct comparison between the groups confirmed significantly greater right hippocampal activation in the older adults (Maguire and Frith, 2003). We believe the current bilateral hippocampal activity evident in the comparison of autobiographical with public events is attributable to the older adults. We also examined the young and older subjects separately with respect to memory remoteness. However, neither group showed any changes in activity associated with remoteness at a threshold of $p<0.001$ (uncorrected), although right (and not left) hippocampal modulation was apparent for both groups at the liberal threshold of $p<0.05$. The two groups did not differ in this regard when directly compared. Therefore, the right hippocampal response to remoteness only emerges as significant in the amalgamated and larger group of subjects. It is perhaps not surprising that small incremental changes in memory age produce effects that are very subtle, but nevertheless consistent across subjects. This may have implications for future fMRI studies planning to examine consolidation/ remoteness in autobiographical memories, suggesting the necessity for a large sample size.

Although activity in the right hippocampus decreases the more remote the autobiographical memories, the gradient of this decrease is over decades. Figure $3 A$ shows that the right hippocampus is still active for memories that are 5 or even 10 years old, and likewise for memories 30 years old, albeit to a much lesser degree. Figure $3 B$ shows that it is only when memories are very remote, $>30$ to 40 years old, that the right hippocampus becomes deactivated and, we assume, not involved. This pattern of findings raises a number of intriguing questions: What is the right hippocampus doing that becomes less relevant over the course of 30 years? Given that we ensured that the recent and remote autobiographical memories were comparable for amount of details recalled (see Materials and Methods), what is different about memories from 60 years ago compared with ones that are, say, 3 years old? Recent and remote memories may differ along lines not detected by our selection process and debriefing. Perhaps the right hippocampus is necessary for the initial processing of the spatial context of an event, given its acknowledged role in spatial memory (O'Keefe and Nadel, 1978; Maguire et al., 1998; Burgess et al., 2002), or recent memories may be more visuoperceptually salient (Pigott and Milner, 1993). Examination of the stimuli did not suggest any differences in the spatial or visuoperceptual details of recent and remote memories. Additional studies explicitly designed to vary both remoteness and also level of detail or other experiential aspects of the memory events will be required to address this issue directly.

The current findings are in agreement with MTT in relation to continuous involvement of the left hippocampus and autobiographical memory. Although protracted involvement of the right hippocampus is also in line with MTT, this theory does not pre- 
dict a parametric decrease in its activity. Although the consolidation theory can embrace the right hippocampal finding, it does not predict significant involvement even after 10 years, nor does the theory hold for the invariant involvement of the left hippocampus. Thus, the current data suggest that both of the two main theories are correct, but only in part.

The MTT and the consolidation theory seem to imply that the posited hippocampal effects are bilateral. However, there is no reason to make this assumption, particularly because there is already evidence that the two medial temporal lobes show relative differences with respect to the types of materials they process: right visuospatial (Smith and Milner, 1981; Abrahams et al., 1997) and left verbal (Frisk and Milner, 1990). The two theories are principally based on findings from patients with bilateral hippocampal/medial temporal damage. If the hippocampi diverge with respect to remoteness, is this supported by data from patients with unilateral lesions? To date, there have been very few studies of remote memory in patients with unilateral temporal lobe damage, and those that have been conducted differ widely in the memory types assessed (autobiographical or public events), tests used, and types of patients examined. Furthermore, damage in these patients was not restricted to the hippocampus. All but one study (Eslinger, 1998) reported unilateral patients to be impaired on tests of remote memory, with either left- or right-sided lesions/epilepsy foci (Bergin et al., 2000; Viskontas et al., 2000) or with only left-sided lesions (Barr et al., 1990; Leplow et al., 1997). In cases in which it was tested, there were no reports of a temporal gradient (Barr et al., 1990; Leplow et al., 1997; Viskontas et al., 2000), although Barr et al. (1990) noted that the patients with a right-temporal lesion performed relatively poorly for recent events.

Patients with semantic dementia (Snowden et al., 1989) have also been reported to have impaired autobiographical memory (Nestor et al., 2002). Their apparently preserved memory for recent rather than remote events in the context of extensive temporal neocortical damage seems to support the consolidation theory, although this is debated (Graham, 1999; Moscovitch and Nadel, 1999; Westmacott et al., 2001; Nestor et al., 2002). In particular, there is mounting evidence that the left hippocampus is implicated early in the course of the disease (Chan et al., 2001; Galton et al., 2001). Given our current results, the semantic dementia findings might simply be explained by the relatively intact right hippocampus processing more recent events, whereas the remote memories, more heavily dependent on the left (and damaged) hippocampus, are impaired.

Lateral asymmetry in remoteness may also interact with the age of patients. Many of those reported with selective bilateral hippocampal damage are older adults (Spiers et al., 2001), as are many semantic dementia cases. Older subjects may be more dependent on the right hippocampus during autobiographical memory retrieval, as suggested by Maguire and Frith (2003). If the right hippocampus is more active for recent events, then damage to this structure in older subjects may be more likely to produce a temporal gradient. Interestingly, Kopelman et al. (1989) found that young amnesic patients showed a relatively flat temporal gradient, whereas older amnesic patients demonstrated a more marked gradient.

\section{Emotion}

Overall, emotional valence and intensity of event memories were not directly correlated with changes in activity. Remoteness and valence were correlated behaviorally for both autobiographical events and public events. Interestingly, they were negatively cor- related for autobiographical events (i.e., more positive memories were more recent) and positively correlated for public events (i.e., recent memories were regarded as more negative). Thus, the right hippocampus and dorsal amygdalas may be responding specifically to recent autobiographical events that have a more positive valence. In a previous neuroimaging study, a preponderance of right hemisphere activity associated with recall of autobiographical events was interpreted as likely attributable to the personal memories being more emotive (Fink et al., 1996), although emotion was not formally manipulated, nor was remoteness of memories. Although the amygdala is often associated with negative emotions such as fear (for review, see Calder et al., 2001), there are recent studies showing that the amygdala may also play a role in processing positive emotions (Baxter and Murray, 2002; Hamann et al., 2002). Although the current data are in line with this view, we interpret them cautiously given that no direct correlation of amygdala activity with emotion was found.

In summary, using a parametric event-related random-effects design, we found lateral asymmetry in the fMRI response of the hippocampi to the remoteness of autobiographical memories. The right hippocampus showed a temporal gradient, whereas the left hippocampus did not. The above design parameters as well as a large number of subjects may be necessary to detect remoteness effects using fMRI. In addition, these effects were evident only for autobiographical and not for public event memories, thus cautioning against using one or the other to generalize about memory remoteness. Although the neural basis of memory is clearly a complex issue, the current results may help to narrow the gulf between neuroimaging and neuropsychological findings, while broadening the scope of theoretical interpretations concerning the role of the hippocampi in remote memory.

\section{References}

Abrahams S, Pickering A, Polkey CE, Morris RG (1997) Spatial memory deficits in patients with unilateral damage to the right hippocampal formation. Neuropsychologia 35:11-24.

Amaral DG (1999) Introduction: what is where in the medial temporal lobe? Hippocampus 9:1-6.

Barr WB, Goldberg E, Wasserstein J, Novelly RA (1990) Retrograde amnesia following unilateral temporal lobectomy. Neuropsychologia 28:243-255.

Baxter MG, Murray EA (2002) The amygdala and reward. Nat Rev Neurosci 3:563-573.

Bergin PS, Thompson PJ, Baxendale SA, Fish DR, Shorvon SD (2000) Remote memory in epilepsy. Epilepsia 41:231-239.

Brett M, Anton JL, Valabregue R, Poline JB (2002) Region of interest analysis using an SPM toolbox. Presented at the 8th International Conference on Functional Mapping of the Human Brain, June 2-6, Sendai, Japan. NeuroImage 16:497.

Burgess N, Maguire EA, Spiers H, O’Keefe J (2001) A temporoparietal and prefrontal network for retrieving the spatial context of life-like events. NeuroImage 14:439-453.

Burgess N, Maguire EA, O’Keefe J (2002) The human hippocampus and spatial and episodic memory. Neuron 35:625-641.

Calder AJ, Lawrence AD, Young AW (2001) Neuropsychology of fear and loathing. Nat Rev Neurosci 2:352-363.

Chan D, Fox NC, Scahill RI, Crum WR, Whitwell JL, Leschziner G, Rossor AM, Stevens JM, Cipolotti L, Rossor MN (2001) Patterns of temporal lobe atrophy in semantic dementia and Alzheimer's disease. Ann Neurol 49:433-442.

Cipolotti L, Shallice T, Chan D, Fox N, Scahill R, Harrison G, Stevens J, Rudge P (2001) Long-term retrograde amnesia ... the crucial role of the hippocampus. Neuropsychologia 39:151-172.

Conway MA, Turk DJ, Miller SL, Logan J, Nebes RD, Meltzer CC, Becker JT (1999) A positron emission tomography (PET) study of autobiographical memory retrieval. Memory 7:679-702.

Eslinger PJ (1998) Autobiographical memory after temporal lobe lesions. Neurocase 4:481-495.

Fink GR, Markowitsch HJ, Reinkemeier M, Bruckbauer T, Kessler J, Heiss 
WD (1996) Cerebral representation of one's own past: neural networks involved in autobiographical memory. J Neurosci 16:4275-4282.

Frisk V, Milner B (1990) The role of the left hippocampal region in the acquisition and retention of story content. Neuropsychologia 28:349-359.

Friston KJ, Holmes AP, Worsley KJ, Frith CD, Frackowiak RS (1995) Statistical parametric maps in functional imaging: a general linear approach. Hum Brain Mapp 2:189-210.

Galton CJ, Patterson K, Graham K, Lambon-Ralph MA, Williams G, Antoun N, Sahakian BJ, Hodges JR (2001) Differing patterns of temporal atrophy in Alzheimer's disease ad semantic dementia. Neurology 57:216-225.

Graham KS (1999) Semantic dementia: a challenge to the multiple-trace theory? Trends Cogn Sci 3:85-87.

Haist F, Bowden Gore J, Mao H (2001) Consolidation of human memory over decades revealed by functional magnetic resonance imaging. Nat Neurosci 4:1139-1145.

Hamann SB, Ely TD, Hoffman JM, Kilts CD (2002) Ecstasy and agony: activation of the human amygdala in positive and negative emotion. Psychol Sci 13:135-141.

Kapur N (1999) Syndromes of retrograde amnesia: a conceptual and empirical synthesis. Psychol Bull 125:800-825.

Kopelman MD, Wilson BA, Baddeley AD (1989) The autobiographical memory interview: a new assessment of autobiographical and personal semantic memory in amnesic patients. J Clin Exp Neuropsychol 11:724-744.

Leplow B, Dierks CH, Lehnung M, Kenkel S, Behrens C, Franks G, Mehdorn M (1997) Remote memory in patients with acute brain injuries. Neuropsychologia 35:881-892.

Maguire EA (2001) Neuroimaging studies of autobiographical event memory. Philos Trans R Soc Lond B Biol Sci 356:1441-1451.

Maguire EA, Frith CD (2003) Aging affects the engagement of the hippocampi during autobiographical memory retrieval. Brain, in press.

Maguire EA, Mummery CJ (1999) Differential modulation of a common memory retrieval network revealed by PET. Hippocampus 9:54-61.

Maguire EA, Burgess N, Donnett JG, Frackowiak RSJ, Frith CD, O’Keefe J (1998) Knowing where, and getting there: a human navigation network. Science 280:921-924.

Maguire EA, Mummery CJ, Büchel C (2000) Patterns of hippocampalcortical interaction dissociate temporal lobe memory subsystems. Hippocampus 10:475-482.

Maguire EA, Henson RNA, Mummery CJ, Frith CD (2001a) Activity in right prefrontal cortex, but not hippocampus, varies parametrically with the increasing remoteness of memories. NeuroReport 12:441-444.

Maguire EA, Vargha-Khadem F, Mishkin M (2001b) The effects of bilateral hippocampal damage on fMRI regional activations and interactions during memory retrieval. Brain 124:1156-1170.

Moscovitch M, Nadel L (1999) Multiple-trace theory and semantic dementia: response to KS Graham. Trends Cogn Sci 3:87-89.

Moscovitch M, Yaschyshyn T, Ziegler M, Nadel L (1999) Remote episodic memory and retrograde amnesia: was Endel Tulving right all along? In: Memory, consciousness, and the brain: the Tallinn Conference (Tulving E, ed), pp 331-345. New York: Psychology.

Murray EA, Bussey TJ (2001) Consolidation and the medial temporal lobe revisited: methodological considerations. Hippocampus 11:1-7.

Nadel L, Moscovitch M (1997) Memory consolidation, retrograde amnesia and the hippocampal complex. Curr Opin Neurobiol 7:217-227.
Nadel L, Samsonovich A, Ryan L, Moscovitch M (2000) Multiple trace theory of human memory: computational, neuroimaging, and neuropsychological results. Hippocampus 10:352-368.

Nestor PJ, Graham KS, Bozeat S, Simons JS, Hodges JR (2002) Memory consolidation and the hippocampus: further evidence from studies of autobiographical memory in semantic dementia and frontal variant frontotemporal dementia. Neuropsychologia 40:633-654.

Niki K, Luo J (2002) An fMRI study on the time-limited role of the medial temporal lobe in long-term topographical autobiographical memory. J Cogn Neurosci 14:500-507.

O'Keefe J, Nadel L (1978) The hippocampus as a cognitive map. Oxford: Oxford UP.

Otten LJ, Henson RN, Rug MD (2002) State-related and item-related neural correlates of successful memory encoding. Nat Neurosci 5:1339-1344.

Pigott S, Milner B (1993) Memory for different aspects of complex visual scenes after unilateral temp. Neuropsychologia 31:1-15.

Rosenbaum RS, Winocur G, Moscovitch M (2001) New views on old memories: re-evaluating the role of the hippocampal complex. Behav Brain Res 127:183-197.

Rugg M (1998) Convergent approaches to electrophysiological and hemodynamic investigations of memory. Hum Brain Mapp 6:394-398.

Ryan L, Nadel L, Keil K, Putnam K, Schnyer D, Trouard T, Moscovitch M (2001) The hippocampal complex and retrieval of recent and very remote autobiographical memories: evidence from functional magnetic resonance imaging in neurologically intact people. Hippocampus 11:707-714.

Sanders HI, Warrington EK (1971) Memory for remote events in amnesic patients. Brain 94:661-668.

Scoville WB, Milner B (1957) Loss of recent memory after bilateral hippocampal lesions. J Neurol Neurosurg Psychiatry 20:11-21.

Smith ML, Milner B (1981) The role of the right hippocampus in the recall of spatial location. Neuropsychologia 19:781-793.

Snowden JS, Goulding PJ, Neary D (1989) Semantic dementia: a form of circumscribed cerebral atrophy. Behav Neurol 2:167-182.

Spiers HJ, Maguire EA, Burgess N (2001) Hippocampal amnesia. Neurocase 7:357-382.

Squire LR (1992) Memory and the hippocampus: a synthesis from findings with rats, monkeys, and humans. Psychol Rev 99:195-231.

Squire LR, Zola-Morgan S (1997) Amnesia, memory and brain systems. Philos Trans R Soc Lond B Biol Sci 352:1663-1673.

Stark CEL, Squire LR (2000) fMRI activity in the medial temporal lobe during recognition memory as a function of study-test interval. Hippocampus 10:329-337.

Viskontas IV, McAndrews MP, Moscovitch M (2000) Remote episodic memory deficits in patients with unilateral temporal lobe epilepsy and excisions. J Neurosci 20:5853-5857.

Westmacott R, Leach L, Freedman M, Moscovitch M (2001) Different patterns of autobiographical memory loss in semantic memory and medial temporal lobe amnesia: a challenge to the consolidation theory. Neurocase 7:37-55.

Zola-Morgan S, Squire LR, Amaral DG (1986) Human amnesia and the medial temporal region: enduring memory impairment following bilateral lesion limited to CA1 field of the hippocampus. J Neurosci 6:29502967. 